\title{
Tracking in 4 dimensions
}

\author{
${ }^{a}$ N Cartiglia* ${ }^{a, b}$ R. Arcidiacono, ${ }^{c} \mathbf{M}$. Boscardin, ${ }^{d}$ G.F. Dalla Betta, ${ }^{a}$ M. Ferrero,,${ }^{c} \mathbf{F}$. \\ Ficorella, ${ }^{a} \mathbf{M}$. Mandurrino, ${ }^{c}$ L. Pancheri, ${ }^{c} \mathbf{G}$. Paternoster, ${ }^{e} \mathbf{H}$. Sadrozinski, ${ }^{e} \mathbf{A}$. \\ Seiden, ${ }^{a}$ A. Staiano, ${ }^{a}$ V. Sola \\ ${ }^{a}$ : INFN, Torino, Italy \\ ${ }^{b}$ : Università Piemonte Orientale, Novara, Italy \\ ${ }^{c}$ : Fondazione Bruno Kessler,Trento, Italy \\ ${ }^{d}$ : Trento University, Trento, Italy \\ ${ }^{e}$ : UCSC, Santa Cruz, USA. \\ E-mail: nicolo.cartigliaecern.ch
}

In this contribution we review the progress towards the development of a novel type of silicon detectors suited for tracking with a picosecond timing resolution, the so called Ultra-Fast Silicon Detectors.

The goal is to create a new family of particle detectors merging excellent position and timing resolution with $\mathrm{GHz}$ counting capabilities, very low material budget, radiation resistance, fine granularity, low power, insensitivity to magnetic field, and affordability. We aim to achieve concurrent precisions of $\sim 10 \mathrm{ps}$ and $\sim 10 \mu \mathrm{m}$ with a $50 \mu \mathrm{m}$ thick sensor.

The first part of this contribution explains the basic concepts of low-gain silicon sensors, while in the following the main results are presented, together with the efforts to make the design radiation resistance.

The European Physical Society Conference on High Energy Physics

5-12 July

Venice, Italy

${ }^{*}$ Speaker. 


\section{The Ultra-Fast silicon Detector (UFSD) design}

The Low Gain Avalanche Detector design is a concept in silicon detector design that merges the best characteristics of standard silicon sensors with the main feature of APDs. The overarching idea is to design silicon detectors with signals that are large enough to assure excellent timing performance, but to keep the gain as low as possible [1, 2, 3, 4].

Charge multiplication in silicon sensors happens when the charge carriers are in electric fields of the order of $E \sim 300 \mathrm{kV} / \mathrm{cm}$. Under this condition the electrons (and to less extent the holes) acquire sufficient kinetic energy that are able to generate additional e/h pairs. A field value of $300 \mathrm{kV} / \mathrm{cm}$ can be obtained by implanting an appropriate charge density that locally generates very high fields $\left(N_{D} \sim 10^{16} / \mathrm{cm}^{3}\right)$. The gain has an exponential dependence on the electric field $N(l)=N_{o} e^{\alpha(E) l}$, where $\alpha(E)$ is a strong function of the electric field and $l$ is the path length inside the high field region. The additional doping layer present at the $n-p$ junction in the LGAD design, Figure 1, generates the high field necessary to achieve charge multiplication.

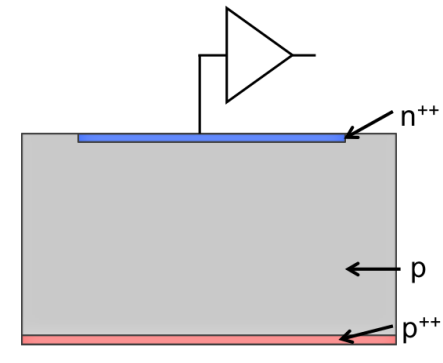

Traditional silicon detector

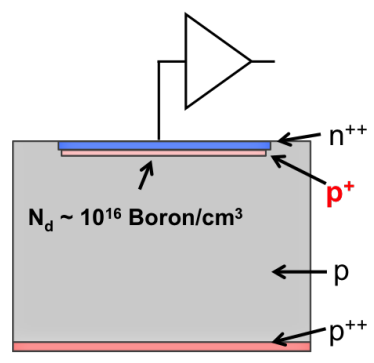

Low gain avalanche detectors

Figure 1: Schematic of a traditional silicon diode (left) and of a Low-Gain Avalanche Diode (right). The additional $p^{+}$layer underneath the $n^{++}$electrode creates, when depleted, a large electric field that generates charge multiplications.

LGAD sensors have the additional advantage of having a large signal $d V / d t$ and therefore being able to measure the signal time accurately. In the following, we will use the name of "UltraFast Silicon Detectors" (UFSD) to indicate LGAD sensors optimized for timing performances.

\section{UFSD timing resolution}

The timing capabilities of UFSD can be explored by modeling the UFSD as a capacitor $\left(C_{D e t}\right)$ with a current source in parallel, readout by a pre-amplifier that shapes the signal. The preamplifier's output is then compared to a fixed threshold $\left(V_{T h}\right)$ to determine the time of arrival. The time resolution $\sigma_{t}$ can be expressed as the sum of several terms: (i) Jitter, (ii) Landau Time Walk (iii) Landau noise due to shape variation, (iv) signal distortion, and (v) TDC binning:

$$
\sigma_{t}^{2}=\sigma_{\text {Jitter }}^{2}+\sigma_{\text {Land.TW }}^{2}+\sigma_{\text {Land. noise }}^{2}+\sigma_{\text {Distortion }}^{2}+\sigma_{T D C}^{2} .
$$

We will assume in the following simplifications: 
- we consider the effect of time walk (see [2] for details) compensated by an appropriate electronic circuit (either Constant Fraction Discriminator or Time over Threshold). With this assumption, the effect of Landau variations in signal amplitude are compensated, but not that of shape variation. This second contribution is indicated as Landau noise $\left(\sigma_{\text {Land. Noise }}^{2}\right)$ in eq. 2.1.

- The $\sigma_{\text {Distortion }}^{2}$ term is due to 2 factors: non uniform weighting field, and non-saturated drift velocity. Both terms are very small in the CMS design since the sensors will always be operated at a bias voltage above $200 \mathrm{~V}$ and the geometry of the pixel, $1 \times 3 \mathrm{~mm}^{2}$ area on a 50-micron thick active substrate assures a very uniform weighting field.

- the contribution of TDC binning to be below $10 \mathrm{ps}$ and therefore negligible.

The UFSD timing resolution is therefore ultimately dominated by two terms: (i) the electronic jitter and (ii) the signal variations due to the non uniform creation of electron-hole pairs along the particle path (we call this effect "Landau noise"). The jitter term is given by the ratio of electronic noise $\mathrm{N}$ and the signal slew rate, $\sigma_{t}($ jitter $)=N /(d V / d t)$, therefore larger signals, for constant noise, have smaller jitter. The Landau noise, instead, depends strongly on the sensor thickness, and moderately on the gain value. Figure 2 [5] shows the variation of these two terms as a function of the UFSD gain, as measured on a Hamamatsu 50-micron thick UFSD sensor: the jitter term, calculated as $\sigma_{t}($ jitter $)=N /(d V / d t)$, decreases constantly with gain while the total time resolution flattens around $\sigma_{t}($ Total $)=30 \mathrm{ps}$ signaling the dominance of the Landau noise contribution.

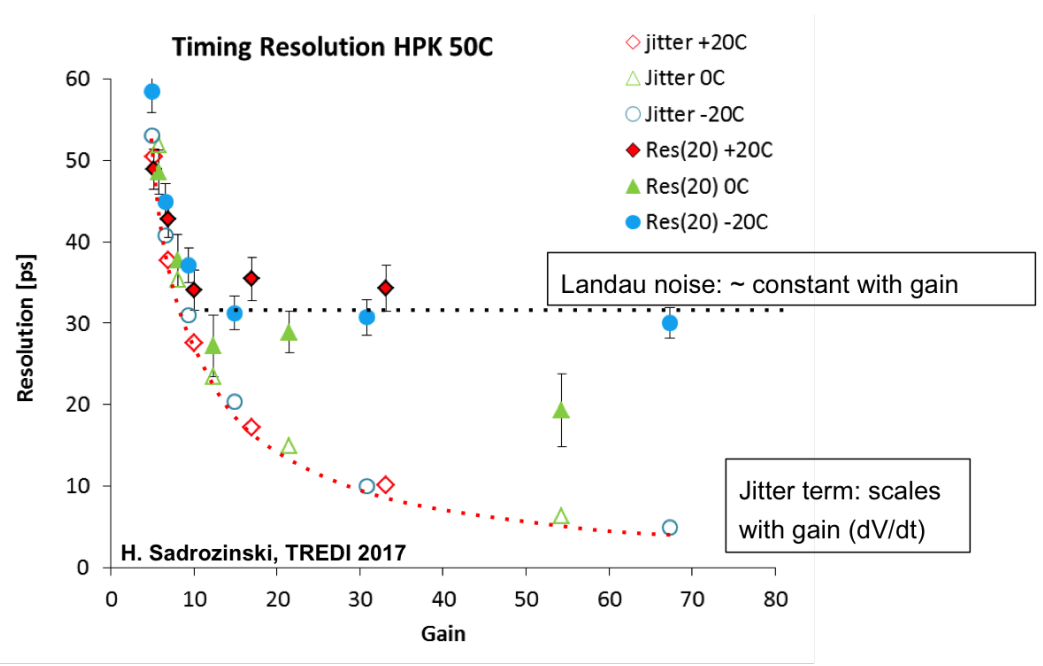

Figure 2: Variation of the jitter and total time resolution as a function of the UFSD gain, as measured on a Hamamatsu 50-micron thick UFSD sensor: the jitter term, calculated as $\sigma_{t}($ jitter $)=N /(d V / d t)$, decreases constantly with gain while the total time resolution flattens around $\sigma_{t}($ Total $)=30 \mathrm{ps}$. 


\section{Results of an extensive irradiation campaign on 50-micron Hamamatsu UFSD sensors}

In this section we focus on the results obtained in the irradiation campaign of 50-micron thick UFSD sensors manufactured by HPK [6]. The sensors have a Boron-doped gain layer which is reduced by irradiation according to the Acceptor removal mechanism". Figure 3 shows the summary of how the gain changes with irradiation, and how the external bias voltage should be increased to compensate for the loss of active doping in the gain layer. All measurements are taken at $-20^{\circ} \mathrm{C}$. As a reference value, the initial bias need to increase from $150 \mathrm{~V}$ to $750 \mathrm{~V}$ to maintain a gain of 10 . The fastest changes are for fluences between $1 \cdot 10^{14}$ and $1 \cdot 10^{15} n_{e q} / \mathrm{cm}^{2}$. Above $1 \cdot 10^{15} \mathrm{n}_{\text {eq }} / \mathrm{cm}^{2}$ the gain is obtained mostly by the field generated by the external bias.

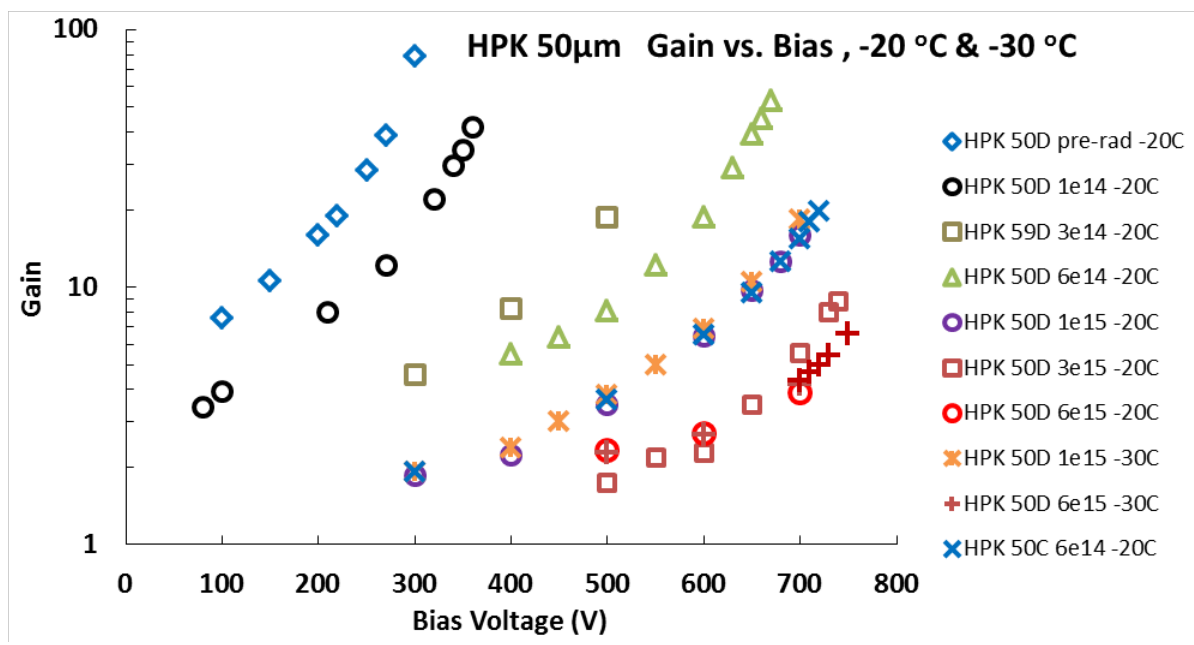

Figure 3: Gain as a function of bias of the LGAD irradiated to the indicated neutron fluences at $-20{ }^{\circ} \mathrm{C}$ and $-30^{\circ} \mathrm{C}$, showing the need for increasing the bias of irradiated sensors to reach adequate gain.

Figure 4 shows the time resolution for the irradiation points presented in Figure 3. The most obvious feature shown by the data is that the only relevant parameter for the time resolution (assuming saturated drift velocity) is the gain value, regardless of the fluence, and that the resolution is indeed getting better for gain up to $\sim 20$. However, in the plot it is possible to identify an additional interesting feature: at low gain there are two different groups, with the first one formed by the lower-fluence data up to $6 \cdot 10^{14} n_{e q} / \mathrm{cm}^{2}$ and the second by higher-fluence data. For a fixed value of gain, for example gain $=5$, the lower-fluence group has a worse time resolution than the higher-fluence group. This fact, which might seems counter-intuitive, is due to the different type of multiplication mechanism in the two groups: below a fluence of $1 \cdot 10^{15} n_{e q} / \mathrm{cm}^{2}$, the multiplication occurs mainly in the gain layer, while for higher fluences the multiplication happens everywhere in the bulk, allowing for the multiplication process to start earlier. This fact leads to two changes in the pulse shape that improve the time resolution: the multiplication is smoother and the rise time decreases. 


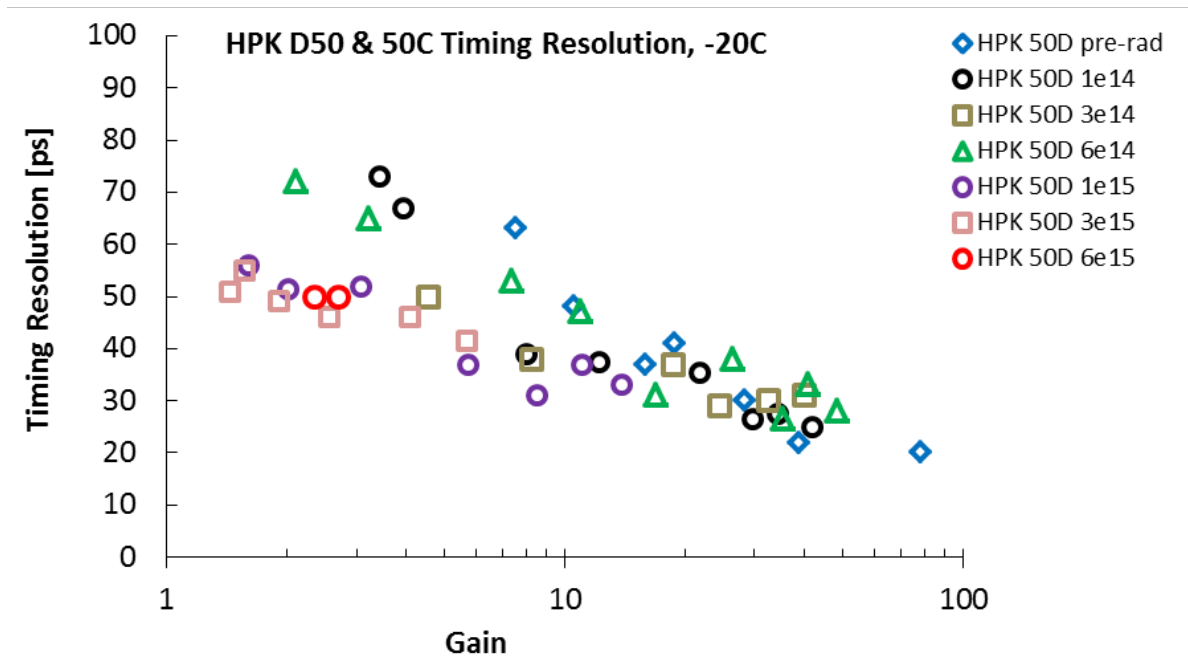

Figure 4: Time resolution evaluated at an optimized CFD fractions vs. gain for the different fluences at -20 ${ }^{o} \mathrm{C}$.

\section{Defect engineering the gain layer: production of UFSD sensors at Fondazione Bruno Kessler}

In spring 2017 the Fondazione Bruno Kessler (FBK, Trento, Italy) completed a production of $50 \mu \mathrm{m}$ thick UFSD with different dopants of the gain layer. Specifically, the gain layer was realized with standard Boron implant as reference in some wafers while other wafers used lowdiffusion Boron, Gallium, and Carbon co-implantation, both with Boron and Gallium. The total production consists of 20 wafers. The use of $\mathrm{C}$ and $\mathrm{Ga}$ dopants has been proposed within the RD50 collaboration [7] in order to mitigate the disappearance of the gain layer due to radiation damage. Carbon reduces the concentration of interstitials available for capturing B atoms during irradiation, and while Gallium reduces the formation of the acceptor-interstitial. The first production batch has been tested in the INFN laboratory in Torino and IV and CV curves measured. C co-implant has the effect of increasing the leakage current both in the $\mathrm{Ga}$ and in the B implant test structures. Breakdown voltage occurs at comparable values, but the Low $\mathrm{C}$ structures have higher $\mathrm{V}_{B D}$ w.r.t. structures without $\mathrm{C}$, both in $\mathrm{Ga}$ and $\mathrm{B}$ doped sensors. The $\mathrm{CV}$ curves, indicate that Carbon coimplantation reduces the number of active dopants, somewhat more in Gallium than in Boron. These results are qualitatively in agreement with the effect of $\mathrm{C}$ co-implantation, of an enhancement of the B (and probably Ga) activation, as observed in [8]. Good uniformity is observed on the various structures tested on wafer prior the final cutting. Detectors are currently being sent to the RD50 colleagues of Jozef Stefan Institute of Ljubljana for the neutron irradiation campaign.

\section{Conclusions}

Ultra Fast Silicon Detectors are quickly becoming a mature technology to be used in high granularity timing sensitive applications. A time resolution of $\sim 30 \mathrm{ps}$ has been achieved at beam tests performed with detectors produced by different manufacturers. Results after exposure to NIEL at 
$6 \cdot 10^{15} n_{e q} / \mathrm{cm}^{2}$, levels to be considered in HL-LHC, look very promising, and further improvements, investigated with new implants of $\mathrm{Ga}$ and $\mathrm{C}$ are currently under test (II FBK run). The interest and soundness of the LGAD technology is well proven by the rapidly spreading commitment of different manufacturers, as CNM, FBK, HPK and more recently Micron Semiconductros ltd [9].

\section{Acknowledgments}

We acknowledge the fundamental contributions coming from the discussions, and active collaboration of the RD50 colleagues. Part of this work has been financed by the European Union's Horizon 2020 Research and Innovation funding program, under Grant Agreement no. 654168 (AIDA-2020) and Grant Agreement no. 669529 (ERC UFSD669529), and by the Italian Ministero degli Affari Esteri and INFN Gruppo V

\section{References}

[1] G. Pellegrini et al, Technology developments and first measurements of Low Gain Avalanche Detectors (LGAD) for high energy physics applications, Nucl.Instrum.Meth. 765 (2014) 24. doi: https://doi.org/10.1016/j.nima.2014.06.008

[2] H. Sadrozinski, A. Seiden and N. Cartiglia, 4-Dimensional Tracking with Ultra-Fast Silicon Detectors, arxiv:1704.08666

[3] N. Cartiglia et al, Design Optimization of Ultra-Fast Silicon Detectors, Nucl.Instrum.Meth. A796, (2015), 141-148. doi: https://doi.org/10.1016/j.nima.2015.04.025

[4] N.Cartiglia et al, Beam test results of a 16 ps timing system based on ultra-fast silicon detectors, Nucl.Instrum.Meth. 850 (2017) 83-88. doi: https:doi.org/10.1016/j.nima.2017.01.021

[5] H.Sadrozinski et al., Timing Measurements on Ultra-Fast Silicon Detectors, 12th Trento Workshop on Advanced Silicon Radiation Detectors, 20 Feb 2017, Trento

[6] Z. Galloway et al., Properties of HPK UFSD after neutron irradiation up to $610^{15} \mathrm{n}_{e q} / \mathrm{cm}^{2}$, arxiv:1707.04961

[7] CERN RD50 collaboration, http://rd50.web.cern.ch/rd50/

[8] Y. Shimizu et al., Impact of carbon co-implantation on boron distribution and activation in silicon studied by atom probe tomography and spreading resistance measurements, Japanese Journal of Applied Physics 55, 026501 (2016)

[9] R. Bates, Simulation and characterisation of low gain avalanche detector for particle physics and synchrotron applications, $11^{\text {th }}$ International Conference on Position Sensitive Detectors, 3-8 Sep 2017, The Open University, Milton Keynes, UK 